\title{
RHEOLOGICAL PROPERTIES OF HEATED CROSS-LINKED WAXY MAIZE STARCH DISPERSIONS
}

\author{
Pedro M. S. Da Silva ${ }^{1}$, J. C. Oliveira ${ }^{1}$, M. A. Rao $^{2, *}$ \\ ${ }^{1}$ Escola Superior de Biotecnologia \\ Rua Dr. Antonio Bernardino de Almeida, 4200 Porto, Portugal. \\ ${ }^{2}$ Department of Food Science and Technology \\ Cornell University, Geneva, NY 14456, USA; "Corresponding author
}

\begin{abstract}
The effect of starch concentration on time-dependent, steady shear, and dynamic shear rheological properties of heated cross-linked waxy maize starch (CWM) starch dispersions was studied. The influence of storage time at $5^{\circ} \mathrm{C}$ on the rheological properties, the applicability of the Cox-Merz rule, and the sol-gel transition of a $5 \%$ (w/w) were also examined. The heated CWM starch dispersions exhibited antithixotropic and shear-thinning with yield stress behaviors. The former behavior increased with both starch concentration and shear rate between $100 \mathrm{~s}^{-1}$ and $400 \mathrm{~s}^{-1}$. As expected, the apparent viscosity at a fixed shear rate and the yield stress increased with starch concentration. Because the Cox-Merz rule was not applicable, the dispersions may be classified as weak gels. Both differential scanning calorimetry and thermorheological data showed that the onset of starch gelatinization temperature was $63^{\circ} \mathrm{C}$.
\end{abstract}

\section{INTRODUCTION}

Starch is naturally present in many foods of plant origin. It is used extensively as a thickening agent in semisolid foods such as sauces, custards, pie fillings, desserts, etc. In comparison to naturally occurring starches, chemically cross-linked starches exhibit better stability to heat, $\mathrm{pH}$, shear and freeze-thaw cycles. These starches are incorporated mainly in canned and frozen foods, and they influence freezing and heating rates, and the rheological properties of the final product (Galliard, 1987).

There have been many studies on the rheological behavior of heated starch dispersions, but here only the studies on CWM dispersions and related studies are 
discussed. Evans and Haisman (1979) and Colas (1986) found the flow behavior of CWM dispersions to be shear thinning with yield stress at $60 \mathrm{~Pa}$ and $25^{\circ} \mathrm{C}$, respectively. Shear thickening (often incorrectly called dilatancy) is not a commonly encountered phenomenon in foods, but has been reported in association with initial stages of gelatinization of CWM (Dail and Steffe, $1990 \mathrm{a}, \mathrm{b}$ ) and other starch dispersions (Bagley and Christianson, 1982; ; Okechukwu and Rao, 1995). Dintzis and Bagley (1995) reported anti-thixotropic behavior in a heated CWM starch dispersion.

Time dependent flow properties of starch dispersions have not been studied extensively. The associated behavior is called either thixotropy or anti thixotropy, depending on either recoverable decrease or increase of viscosity with time under constant shear rate conditions, respectively. This behavior is usually related to either the breakdown (thixotropy) or buildup (anti thixotropy) of structures in a dispersion, although other phenomena may originate it in concentrated dispersions (Boersma et al., 1991). The Weltman model (1943) describes thixotropic flow behavior of a fluid:

$$
\sigma=\mathrm{A}-\mathrm{B} \log \mathrm{t}
$$

where, is $\sigma$ shear stress $(\mathrm{Pa}), \mathrm{t}$ is time, and $\mathrm{A}$ and $\mathrm{B}$ are constants that characterize a fluid's time dependent behavior. Although the model has been used to describe thixotropic behavior of foods (Paredes et al., 1988), it is also suitable to describe antithixotropic behavior. Steady shear behavior of fluids that exhibit yield stress is often modeled using the Herschel-Bulkley (HB) equation:

$$
\sigma=\sigma_{0}+K_{H} \gamma^{n_{H}}
$$

where, $\sigma_{\mathrm{o}}$ is the yield stress $(\mathrm{Pa}), K_{H}$ is consistency index $(\mathrm{Pa} \mathrm{s})$, and $\eta_{H}$ is flow behavior index (dimensionless). Christianson and Bagley (1984) used the Casson model to estimate yield stresses of dispersions of unmodified starch dispersions. However, because the $\mathrm{HB}$ model has been used in processing and handling applications, it was used in the present study.

Dynamic rheological properties $\left(G^{\prime}, G^{\prime \prime}\right.$, and $\left.\eta^{*}\right)$ can be used to quantitatively describe the viscoelastic nature of a material and comparison with steady shear rheological properties may provide additional insight on its structure (Ross-Murphy, 1984). Cox and Merz (1958) proposed an empirical method to correlate dynamic and steady shear rheological data at equal values of frequency $\left(\omega, \operatorname{rad~s}^{-1}\right)$ and shear rate $(\gamma$, $\mathrm{s}^{-1}$ ), respectively:

$$
\mid \eta * \eta^{*}=\eta_{\omega=} \gamma
$$

where, $\eta^{*}$ is the complex viscosity ( $\mathrm{Pa} \mathrm{s}$ ) and $\eta_{a}$ is the apparent viscosity $(\mathrm{Pa} \mathrm{s})$. The Cox-Merz rule was found to be applicable for several synthetic and biopolymer dispersions, but not for biopolymer dispersions with either hyperentanglements or aggregates (Lopes da Silva and Rao, 1992) and complex food systems (Bistany and Kokini, 1983). When the Cox-Merz rule is followed, it may be used to predict the 
steady shear properties of a material from the dynamic rheological properties obtained without extensive alteration of structures.

The objectives of this study were to examine the: (1) time-dependent $\left(\eta_{\mathrm{a}}\right.$-time at fixed $\gamma$ ) and steady flow $(\sigma-\gamma)$, and frequency dependent dynamic shear ( $G^{\prime}$ and $\left.\mathrm{G}^{\prime \prime}-\omega\right)$ rheological properties of heated aqueous CWM starch dispersions measured at $20^{\circ} \mathrm{C}$, (2) influence of different starch concentrations, $3 \%, 4 \%, 5 \%$, w/w, on the properties, (3) effect of time of storage at $5{ }^{\circ} \mathrm{C}$ on the properties of $5 \%$ dispersions, (4) applicability of the Cox-Merz rule to predict steady shear properties from dynamic shear data, and (5) sol-gel transition of a 5\% dispersion using differential scanning calorimetry and temperature sweep rheology data.

\section{MATERIALS}

CWM starch (National Starch and Chemical Company, Bridgewater, NJ, product designation: COL FLO 67, lot \# TBK 3886) dispersions in distilled water were prepared in an insulated jacketed stainless steel vessel $(\approx 350 \mathrm{ml})$ by heating at $80^{\circ} \mathrm{C}$ under continuous mild agitation. A circulating water bath (Isotemp, 901, Fisher Scientific, Pittsburgh, PA) was used to control the sample temperature. A type T thermocouple connected to a digital panel meter (Omega Engineering, Inc., Stamford, CT) placed inside the dispersions was used to monitor temperature.

To prepare a $3 \%$ starch dispersion, $3.0 \mathrm{~g}$ of starch and $97.0 \mathrm{~g}$ of water were weighed. A small amount, about $2 \mathrm{~mL}$, of water was added to the starch sample to create a slurry that was added to the remaining quantity of water at $80^{\circ} \mathrm{C}$. Samples of the heated starch dispersion were collected for rheological tests after $3.6 \mathrm{ks}$ and immersed in ice cold water to arrest changes in the sample. Two replicates of the starch concentration were prepared and the procedure was repeated with $4 \%$ and $5 \%$ starch concentrations. With the $5 \%$ starch dispersions, the effect of storage time at $5^{\circ} \mathrm{C}: 0,1$, 3 , and 5 days on rheological properties was investigated that corresponded to data collection on day 1 , day 2 , day 4 , and day 6 , respectively.

\section{METHODS}

\section{Rheological measurements}

A Carri-Med CSL 100 Controlled-Stress Rheometer (TA Instruments, New Castle, DE) together with a $6 \mathrm{~cm}$ dia., $2^{\circ}$ angle, aluminum cone and a solvent trap to minimize moisture loss through evaporation were used to obtain the steady shear and dynamic rheological data.

To determine the time-dependent behavior, each sample at $20^{\circ} \mathrm{C}$ was submitted to one of the constant shear rates: $50 \mathrm{~s}^{-1}, 100 \mathrm{~s}^{-1}, 200 \mathrm{~s}^{-1}, 300 \mathrm{~s}^{-1}$, and $400 \mathrm{~s}^{-1}$ for a period of $1200 \mathrm{~s}$. The steady shear data were obtained by applying increasing shear stresses $(0.01$ to $15.0 \mathrm{~Pa}$ for $3 \%$ dispersions, 0.01 to $75 \mathrm{~Pa}$ for $4 \%$ dispersions, and 0.01 to $150 \mathrm{~Pa}$ for $5 \%$ dispersions) during a $300 \mathrm{~s}$ period, and the shear stresses were applied in the reverse order during the same period of time. The dynamic rheological data were 
obtained over the frequencies: 0.62 to $62.83 \mathrm{rad} \mathrm{s}^{-1}$ applying a shear stress of $2 \mathrm{~Pa}$ to $3 \%$ dispersions, $4.5 \mathrm{~Pa}$ to $4 \%$ dispersions, and $5 \mathrm{~Pa}$ to $5 \%$ dispersions. The applied stresses were ascertained to be within the linear viscoelastic range. In order to study the influence of storage, rheological data were obtained on $5 \%$ dispersions stored for 0,1 , 3 , and 5 days at $5^{\circ} \mathrm{C}$. In each experiment, a fresh starch dispersion sample was used; after it was loaded and the rheometer plate was rammed up, a $900 \mathrm{~s}$ rest period was allowed to restore structures that might have been disturbed.

The Weltman model (Equation 1) and the Herschel-Bulkley model (Equation 2), were used to describe time-dependent and steady shear flow data, respectively. Magnitudes of the two models's parameters were determined by non-linear regression using the ZXSSQ routine (IMSL Inc., Houston, TX) based on the Levenberg-Marquardt algorithm. The accessory subroutines were written in FORTRAN 77.

\section{Sol-gel transition data}

Dynamic rheological data during the sol-gel transition of a $5 \%$ starch dispersion were obtained using a $40 \mathrm{~mm}$ diameter parallel plate, gap width between plates $500 \mu \mathrm{m}$, $5 \mathrm{~Hz}$, as the sample was heated from 30 to $90^{\circ} \mathrm{C}$ at $2^{\circ} \mathrm{C} / \mathrm{min}$. With the larger gap parallel plate system, it was possible to place a thin layer of paraffin oil at the circumference to eliminate moisture loss by evaporation. In contrast, with a cone-plate system with a $52 \mu \mathrm{m}$ gap, the paraffin oil penetrated the starch dispersion due to capillary suction. Sol-gel transition was also investigated with a differential scanning calorimeter (DSC) (model 2910, TA Instruments, Inc.) using $10 \mu \mathrm{m}$ samples in hermetically sealed pans that were heated from 20 to $120^{\circ} \mathrm{C}$ at a rate of $5^{\circ} \mathrm{C} / \mathrm{min}$. The sol-gel transition rheology and DSC experiments were replicated four times.

\section{RESULTS AND DISCUSSION}

\section{Time-dependent flow behavior}

The CWM starch dispersions exhibited a pronounced dependency on shear rate and concentration. While the antithixotropic behavior was barely discernible at $50 \mathrm{~s}^{-1}$ and $100 \mathrm{~s}^{-1}$, it was clearly discernible at the higher shear rates studied so that the degree of the antithixotropic behavior of the dispersion appears to have increased with shear rate. Apparent viscosity versus shearing time data of $5 \%$ gelatinized dispersions at constant shear rates between 100 and $400 \mathrm{~s}^{-1}$ are shown in Figure 1. For the CWM dispersions studied, the antithixotropic data were very well defined at $200 \mathrm{~s}^{-1}$ and amenable to reliable analysis, and less so at lower and higher shear rates. Most of the increase in apparent viscosity occurred within about $400 \mathrm{~s}$ after shearing was initiated. At the highest shear rate $400 \mathrm{~s}^{-1}$, after the initial increase in apparent viscosity increase, a gradual decrease was observed. However, this decrease might have been due to the loss of a small amount of sample as a result of high centrifugal forces in the cone-plate gap. 


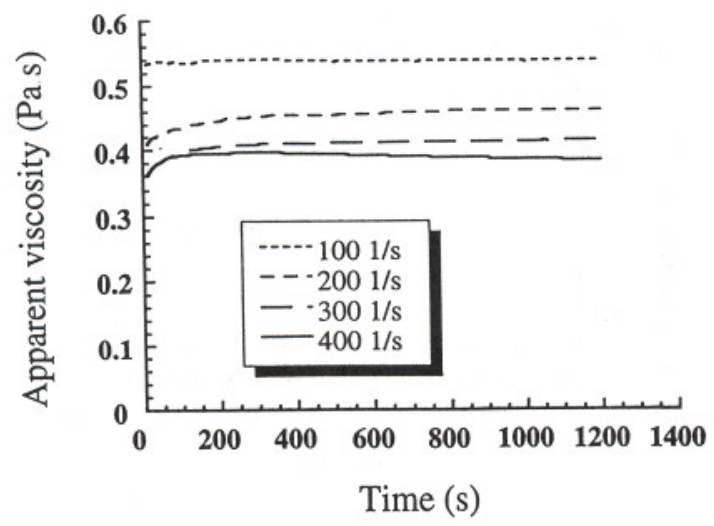

Figure 1. Apparent viscosity, at constant shear rates between 100 and $400 \mathrm{~s}^{-1}$, of a $5 \%$ gelatinized cross-linked waxy maize starch dispersion increased with shearing time with most of the increase taking place in the first $400 \mathrm{~s}$. Data taken at $20^{\circ} \mathrm{C}$.

The CWM starch concentration also had a positive effect on the magnitude of the antithixotropic behavior. The apparent viscosity at $200 \mathrm{~s}^{-1}$ versus shearing time data of dispersions of 3, 4 and $5 \%$ starch concentrations are shown in Figure 2. As starch concentration increased, not only the initial value of apparent viscosity increased significantly but also the rate of increase of the apparent viscosity was higher, i.e., there was an increase in degree of the antithixotropic phenomena. The Weltman model (Equation 1) described well some of the time-dependent data with correlation coefficients greater than 0.999 (Table 1). In Table 1, the model parameters for the data at $400 \mathrm{~s}^{-1}$ of all the dispersions, at $300 \mathrm{~s}^{-1}$ of the $3 \%$ and $4 \%$ starch dispersions, and at $50 \mathrm{~s}^{-1}$ of the $5 \%$ dispersion are not shown due to lack of reproducibility. In Table 1, positive values of the constant $B$ indicate antithixotropic behavior and negative values, such as that of the $5 \%$ dispersion at a shear rate of $100 \mathrm{~s}^{-1}$, indicate thixotropic behavior.

The apparent viscosity at $200 \mathrm{~s}^{-1}$ versus shearing time data of a $5 \%$ dispersion as a function of storage time are shown in Figure 3. Although the average apparent viscosities of the stored samples were different from each other, the shape of the curves remained the same. Storage time up to five days did not seem to have significantly influenced the antithixotropic behavior.

\section{Steady shear data}

Shear stress versus shear rate data of the $3 \%, 4 \%$, and $5 \%$ starch dispersions are shown in Figure 4. Each curve represents the average of three replicates, the down curve (decreasing shear stresses) having been chosen because of better reproducibility. 


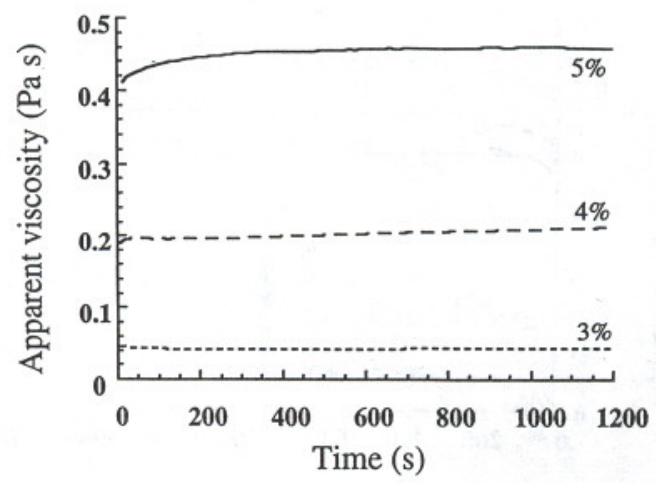

Figure 2. Apparent viscosity at $200 \mathrm{~s}^{\mathbf{- 1}}$ of cross-linked waxy maize starch dispersions at different concentrations $(3,4$ and $5 \%)$ increased with shearing time and starch concentration. Data taken at $20^{\circ} \mathrm{C}$.

Table 1. Weltman equation parameters ${ }^{\mathrm{a}}$ for gelatinized cross-linked waxy maize starch dispersions.

\begin{tabular}{lccccc}
\hline & \multicolumn{5}{c}{ Shear rate $\left(\mathrm{s}^{-1}\right)$} \\
Parameter & Concentration $(\mathrm{w} / \mathrm{w}, \%)$ & 50 & 100 & 200 & 300 \\
\hline \multirow{3}{*}{ A } & 3 & $6.97 \times 10^{-2}$ & $5.76 \times 10^{-2}$ & $4.22 \times 10^{-2}$ & n. d. \\
& 4 & $2.97 \times 10^{-1}$ & $2.08 \times 10^{-1}$ & $1.72 \times 10^{-1}$ & $9.54 \times 10^{-2}$ \\
& 5 & n. d. & $5.39 \times 10^{-1}$ & $3.88 \times 10^{-1}$ & $3.55 \times 10^{-1}$ \\
\hline \multirow{2}{*}{ B } & 3 & $1.58 \times 10^{-3}$ & $5.71 \times 10^{-4}$ & $1.66 \times 10^{-5}$ & n. d. \\
& 4 & $3.15 \times 10^{-3}$ & $2.07 \times 10^{-3}$ & $5.29 \times 10^{-3}$ & $7.83 \times 10^{-3}$ \\
& 5 & n. d. & $-8.30 \times 10^{-5}$ & $1.06 \times 10^{-2}$ & $8.79 \times 10^{-3}$ \\
\hline
\end{tabular}

a'Correlation coefficient $>0.999$ in all cases

n.d. denotes not determined due to poor reproducibility of data.

Positive values of B indicate antithixotropic behavior and the single negative value indicates thixotropic behavior.

Samples of the $3 \%$ dispersions were submitted to lower shear rates because of the tendency of the sample to 'spread' out of the gap at high shear rates and thus artificially lowering the apparent viscosity.

Figure 5 shows the effect of storage time at $5^{\circ} \mathrm{C}$ on the flow curves. The Herschel-Bulkley model described well the rheological data obtained at all concentrations and storage times. The Herschel-Bulkley model parameters as affected by concentration and storage time are summarized in Table 2 and Table 3 , respectively. 


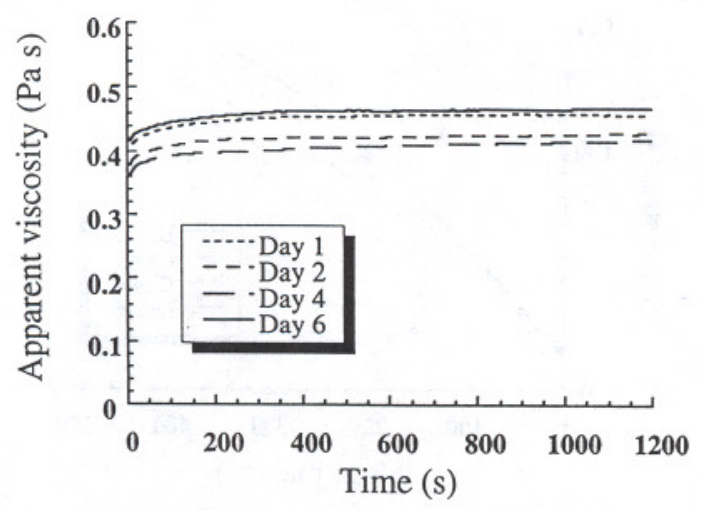

Figure 3. Influence of storage of gelatinized $5 \%$ cross-linked waxy maize starch dispersions at $5^{\circ} \mathrm{C}$ on the apparent viscosity at $200 \mathrm{~s}^{-1}$ as a function of shearing time. The days on which data were taken are shown. Data taken at $20^{\circ} \mathrm{C}$.

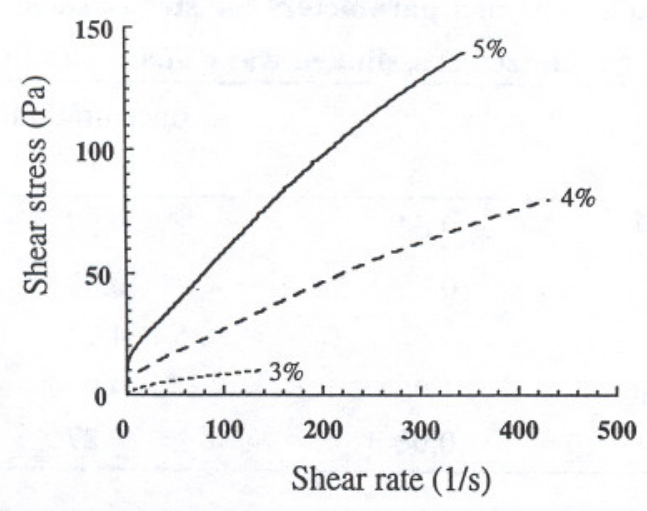

Figure 4. Shear stress versus shear rate curves of gelatinized 3, 4 and $5 \%$ crosslinked waxy maize starch dispersions. With the $3 \%$ dispersion, data at high shear rates could not be obtained due to loss of sample from the cone-plate geometry. Data taken at $20^{\circ} \mathrm{C}$.

Increase in starch concentration, as would be expected, increased the apparent viscosity (Table 2) while storage time had an antagonistic effect (Table 3) on apparent viscosity. The effect of concentration and storage time on the apparent viscosity was not reflected in the trends of $K_{H}$ and $\eta_{H}$ due to the coupling of the values of the two parameters. We note that in general the yield stress increased with concentration (Table 2) and decreased with storage time (Table 3 ). 


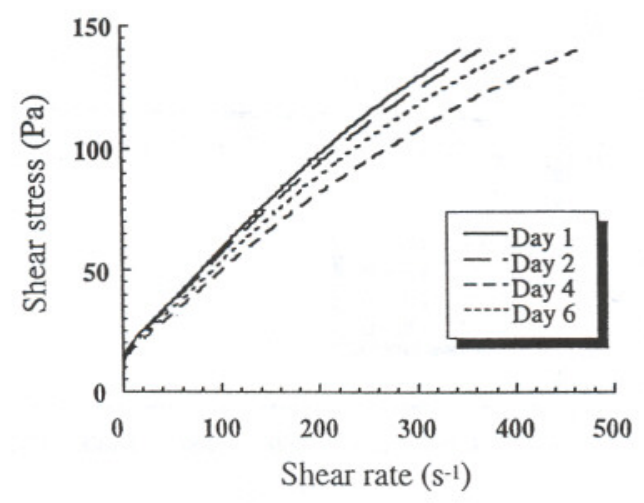

Figure 5. Influence of storage of gelatinized 5\% cross-linked waxy maize starch dispersions at $5^{\circ} \mathrm{C}$ on the shear stress versus shear rate curves. The days on which data were taken are shown. Data taken at $20^{\circ} \mathrm{C}$.

Table 2. Herschel-Bulkley model parameters for steady shear data and apparent viscosity at $100 \mathrm{~s}^{-1}$ of gelatinized crosslinked waxy maize starch dispersions.

Concentration (w/w, \%)

\begin{tabular}{lccl} 
Parameter & 3 & 4 & 5 \\
\hline$K_{H}\left(\mathrm{~Pa} \mathrm{~s}^{\mathrm{n}}\right)$ & 0.64 & 0.57 & 0.92 \\
$\eta_{H}$ & 0.55 & 0.81 & 0.86 \\
$\sigma_{0}(\mathrm{~Pa})$ & 0 & 4.32 & 11.6 \\
Correlation coefficient & 1.00 & 0.999 & 0.999 \\
$\eta_{a} @ 100 s^{-1}(\mathrm{~Pa} \mathrm{~s})$ & 0.08 & 0.27 & 0.59 \\
\hline
\end{tabular}

Table 3. Herschel-Bulkley model parameters for steady shear data and apparent viscosity at $100 \mathrm{~s}^{-1}$ of $5 \%, w / w$ dispersions after different storage times.

Storage time at $5^{\circ} \mathrm{C}$ (days)

\begin{tabular}{lclll} 
Parameter & 0 & 1 & 3 & 5 \\
\hline$K_{H}\left(\mathrm{~Pa} \mathrm{~s}^{\mathrm{n}}\right)$ & 1.24 & 1.17 & 1.12 & 1.13 \\
$n_{H}$ & 0.80 & 0.80 & 0.79 & 0.80 \\
$\sigma_{0}(\mathrm{~Pa})$ & 10.28 & 10.44 & 8.56 & 9.24 \\
\multicolumn{2}{l}{ Correlation coefficient 0.999} & 0.999 & 0.999 & 0.999 \\
$\eta_{a} @ 100 s^{-1}(\mathrm{~Pa} \mathrm{~s})$ & 0.59 & 0.57 & 0.50 & 0.53 \\
\hline
\end{tabular}




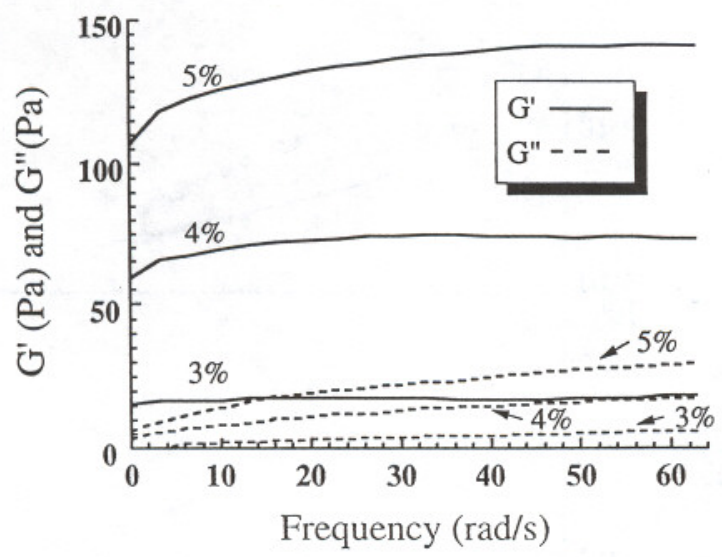

Figure 6. Magnitudes of storage ( $\left.G^{\prime}\right)$ and loss moduli ( $\left.G^{\prime \prime}\right)$ vs. frequency of gelatinized 3, 4 and 5\% cross-linked waxy maize starch dispersions. Data taken at $20^{\circ} \mathrm{C}$.

\section{Dynamic rheological data}

The dynamic rheological properties $G^{\prime}$ and $G^{\prime \prime}$ versus frequency of 3, 4, and $5 \%$ gelatinized CWM starch dispersions are shown in Figure 6. With all the dispersions, values of $G^{\prime}$ were much higher than those of $G^{\prime \prime}$, so that the dispersions exhibited strong gel-like behavior over the studied range of frequencies. Both $G^{\prime}$ and $G^{\prime \prime}$ increased with concentration and showed a relatively weak dependence on frequency at low values of this variable. This dependency was more pronounced in dispersions of higher starch concentrations. At a specific starch concentration, the nearly constant value of $\mathrm{G}^{\prime}$ at high frequencies is often called the plateau modulus, $G_{0}$. Storage time had a very small effect on $G^{\prime \prime}$, but values of $G^{\prime}$ showed small fluctuations of unknown origin (not shown here).

In order to examine the applicability of the Cox-Merz rule (Equation 3), magnitudes of apparent viscosity were plotted against shear rate, and those of complex viscosity $\left(\eta^{*}\right)$ and dynamic viscosity $\left(\eta^{\prime}\right)$ were plotted against frequency (Figure 7 ). In the range of concentrations studied, the Cox-Merz rule was not to applicable to gelatinized CWM starch dispersions (Figure 7), but the dynamic viscosity provided reasonable estimates of the apparent viscosity of the $3 \%$ and $4 \%$ dispersions. The failure of Cox-Merz rule to correlate the steady and dynamic shear data also reflects the gel-like nature of the gelatinized CWM starch dispersions.

\section{Sol-gel transition}

Figure 8 contains the sol-gel transition data obtained using both the Carri-Med CSL 100 rheometer and a TA Instruments DSC 2910 differential scanning calorimeter. The 


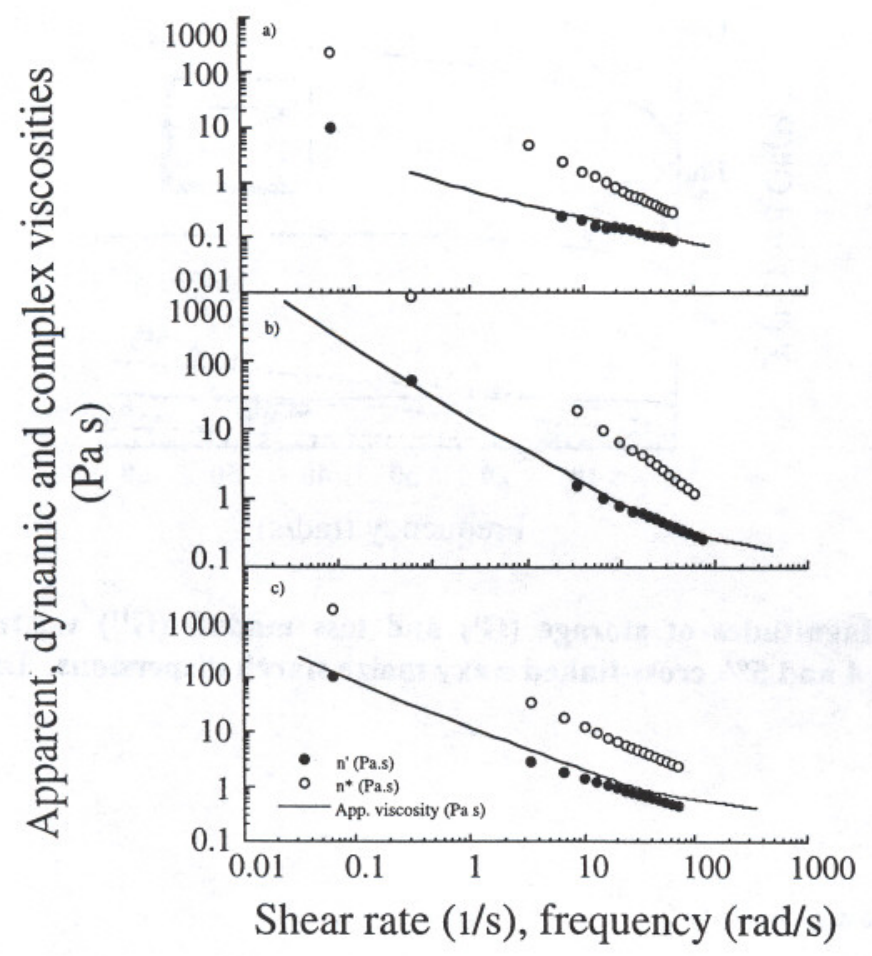

Figure 7. Cox-Merz plots of gelatinized cross-linked waxy maize starch dispersions, from top to bottom, 3\%, 4\%, and 5\%, respectively. Apparent viscosity-shear rate data are shown as lines. Complex viscosity (open circles) and dynamic viscosity (closed circles) are plotted as a function of oscillatory frequency $\left(\operatorname{rad~s}^{-1}\right)$. Data taken at $20^{\circ} \mathrm{C}$.

dynamic properties curves are the average data obtained in four different experiments. Both rheological tests and DSC data shows a gelatinization onset at about $63^{\circ} \mathrm{C}$. Above this temperature, $G^{\prime}$ increased significantly while $G^{\prime \prime}$ remained almost constant. The change in $G^{\prime}$ is a better indicator of the gradual transition from a sol to a gel. The DSC data indicated completion of gelatinization at about $72^{\circ} \mathrm{C}$, but the rheology data indicate further continued increase in $\mathrm{G}^{\prime}$ up to about $85^{\circ} \mathrm{C}$ due starch granule swelling and then a slight decrease probably due to decrease in granule size. From these results, it can be inferred that the CWM dispersions maintained at $80^{\circ} \mathrm{C}$ for 1 hour for rheological measurements were indeed fully gelatinized.

\section{CONCLUSIONS}

Gelatinized CWM starch dispersions exhibited antithixotropic and shear thinning with yield flow behavior. The antithixotropic behavior was well defined at $200 \mathrm{~s}^{-1}$ and 


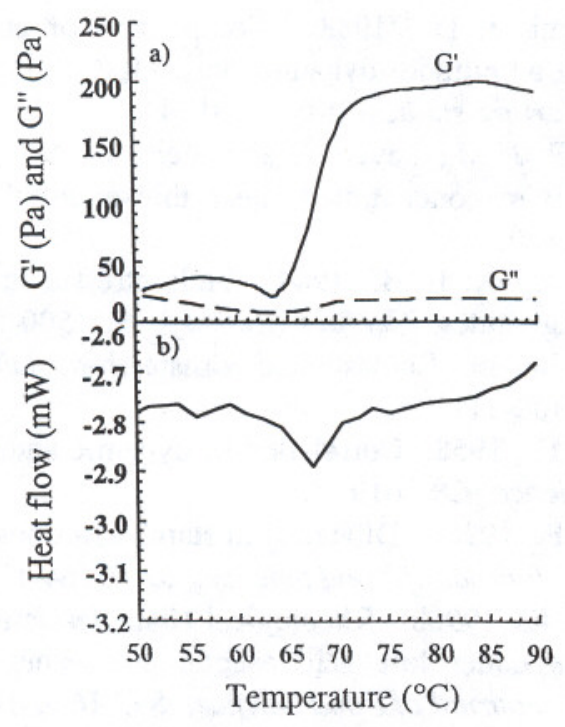

Figure 8. Sol-gel transition data obtained on a $5 \%$ cross-linked waxy maize starch dispersion: (a) storage ( $\left.G^{\prime}\right)$ and loss moduli (G") at $5 \mathrm{~Hz}$ as a function of temperature, and (b) DSC endotherm.

could be described by the Weltman model that was developed to describe thixotropic behavior. Dispersions stored at $5^{\circ} \mathrm{C}$ also showed time-dependent flow behavior. The Hershel-Bulkley model described well the steady shear flow data. Dynamic rheological data of the dispersions revealed that the storage modulus, $\mathrm{G}^{\prime}$, was always higher than the loss modulus, G", and both increased with starch concentration. The Cox-Merz rule was not applicable to the dispersions studied, reflecting their heterogeneous structure. Both rheological and DSC data indicated that the onset of gelatinization of CWM starches was about $63^{\circ} \mathrm{C}$.

\section{ACKNOWLEDGMENTS}

We thank the Junta Nacional de Investigação Científica e Tecnológica (JNICT), Lisbon, Portugal, for a research scholarship to author da Silva, and the National Starch and Chemical Company for donation of starch samples.

\section{REFERENCES}

Bagley, E. B. and Christianson, D. D. 1982. Swelling capacity of starch and its relationship to suspension viscosity: Effect of cooking time, temperature and concentration. Journal of Texture Studies. 13: 115-126. 
Bistany, K. 1. and Kokini, J. L. 1983. Comparison of steady shear rheological properties and small amplitude dynamic viscoelastic properties of fluid food materials. Journal of Texture studies. 14: 113-124.

Boersma, W. H., Baets, P. J. M., Laven, J. and Stein, H. N. 1991. Time-dependent behavior and wall slip in concentrated shear thickening dispersions. Journal of Rheology. 35: 1093-1120.

Christianson, D. D. and Bagley, E. B. 1984. Yield stresses in dispersions of swollen deformable cornstarch granules. Cereal Chemistry. 61: 500-503.

Colas, B. 1986. Flow behavior of crosslinked cornstarches. Lebensmittel Wissenschaft und Technology. 19: 308-311.

Cox, W. P. and Merz, E. H. 1958. Correlation of dynamic and steady-flow viscosities. Journal of Polymer Science. 28: 619-622.

Dail, R. V. and Steffe, J. F. 1990a. Dilatancy in starch solutions under low acid aseptic processing conditions. Journal of Food Science. 55: 1764-1765.

Dail, R. V. and Steffe, J. F. 1990b. Rheological characterization of crosslinked waxy maize starch solutions under low acid aseptic processing conditions using tube viscometry techniques. Journal of Food Science. 55: 1660-1665.

Dintzis, F. R. and Bagley, E. B. 1995. Shear-thickening and transient flow effects in starch solutions. Journal of Applied Polymer Science. 56: 637-640.

Evans, I. D. and Haisman, D. R. 1979. Rheology of gelatinized starch suspensions. Journal of Texture Studies. 10: 347-370.

Galliard, T. 1987. Starch Availability and Utilization. In: Starch: Properties and Potential. Galliard, T. ed. Critical Reports on Applied Chemistry, Blackwell Scientific Publications, London. p. 1-15.

Lopes da Silva, J. A. L. and Rao, M. A. 1992. Viscoelastic Properties of Food Gum Dispersions. In: Viscoelastic Properties of Foods. Rao, M. A. and Steffe, J. F. eds. Elsevier Applied Science Publishers, London. p. 285-316.

Okechukwu, P. E. and Rao, M. A. 1995. Influence of granule size on viscosity of cornstarch suspension. Journal of Texture Studies. 26: 501-516.

Paredes, M. D. C., Rao, M. A., and Bourne, M. C. 1988. Rheological characterization of salad dressings. 1. Steady shear, thixotropy and effect of temperature. Journal of Texture Studies. 19: 247-258.

Ross-Murphy, S. B. 1984. Rheological Methods. In: Biophysical Methods in Food Research. Chan, H. W.-S. ed. Blackwell Scientific, London. p. 138-199.

Weltman, R. N. 1943. Breakdown of thixotropic structure as a function of time. Journal of Applied Physics. 14: 343-350. 